\title{
Suplementos nutricionales y piel
}

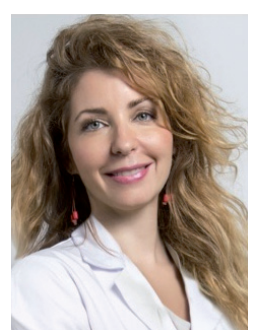

Elena González-Guerra

Profesora asociada

de Dermatología

Médico-Quirúrgica y Venereología

(Ciencias de la Salud).

Departamento de Medicina.

Universidad Complutense

de Madrid.

Médico adjunto del Servicio

de Dermatología.

Hospital Universitario Clínico

San Carlos. Madrid.

Directora del Máster

en Dermofarmacia

y Formulación Cosmética.

Universidad Internacional

de La Rioja (UNIR).

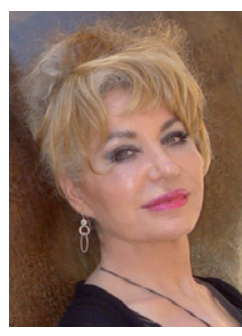

Aurora Guerra-Tapia

Profesora titular de Dermatología.

Departamento de Medicina.

Facultad de Medicina.

Universidad Complutense

de Madrid.

Exjefa de la Sección

de Dermatología.

Hospital Universitario

12 de Octubre. Madrid.

Directora del Máster

en Dermofarmacia

y Formulación Cosmética.

Universidad Internacional

de La Rioja (UNIR).

\author{
"Come poco y cena menos, \\ que la salud de todo el cuerpo \\ se fragua en la oficina del estómago». \\ Miguel de Cervantes Saavedra \\ (1547-1616).
}

\section{INTRODUCCIÓN}

Todo ser vivo necesita un aporte de energía para sobrevivir. Cualquier elemento ingerido con esa finalidad se considera un alimento, y el que bioquímicamente es esencial se denomina nutriente. El proceso biológico llevado a cabo por animales y plantas que implica la asimilación de los nutrientes recibe el nombre de $n u$ trición. Nos queda por definir en todo este proceso un elemento opcional que puede complementarlo: los suplementos nutricionales, que son aquellas fuentes concentradas de nutrientes o de otras sustancias con efecto fisiológico nutricional que se añaden a la dieta.

Por cierto, y a propósito de dieta (del griego $\Delta \iota \alpha \iota \tau \alpha$ [diaita] y del latín diaeta), debemos recordar que, en su primera acepción, el término hace referencia al régimen de vida que incluye a los alimentos y a los ejercicios físicos. En la actualidad, sin embargo, hablamos de dieta cuando hacemos una alimentación metódica basada en el uso preferente de algunos alimentos o en la exclusión de otros. Este concepto terapéutico del término dieta aparece por primera vez en la lengua castellana medieval en el siglo XIII, potenciándose en la actualidad por modas o creencias; así, la dieta vegetariana, vegana, proteica o paleolítica, entre otras. A su vez, en las últimas décadas, el uso de suplementos nutricionales ha experimentado un importante auge. De este fenómeno han surgido nuevos términos, incorporados al vocabulario del mundo sanitario tanto en los ambientes académicos como divulgativos. 
Así, un compuesto nutracéutico se puede definir como un suplemento nutricional compuesto por una sustancia natural bioactiva concentrada, presentado en una matriz no alimenticia (píldoras, cápsulas, polvo, etc.), que normalmente se encuentra en los alimentos y que, tomada en dosis superior a la existente de forma natural, tiene un presumible efecto favorable sobre la salud, mayor que el que podría tener el simple alimento.

Cuando la intención es actuar sobre la piel y el cabello, se habla de nutricosméticos, basados en el concepto de que la clave para mejorar el aspecto y la belleza exterior se puede conseguir buscando la salud global, es decir, la «belleza desde el interior». Sus efectos pueden ejercerse con diversos fines como fotoprotección, antioxidación, antiinflamación, o modulación de la respuesta inmunitaria, de las reacciones enzimáticas o de las señalizaciones celulares.

El debate, la controversia sobre la idoneidad de los suplementos nutricionales para la piel y el cabello, ha surgido con opiniones a favor y en contra, emitiéndose juicios de parte de influenciadores de las redes sociales, de los ambientes económicos, de medios publicitarios, de los defensores de la calidad de vida, del autocuidado, etc.

\section{PERO... ¿QUÉ DICE LA CIENCIA} AL RESPECTO?

Desde el punto de vista científico, los suplementos nutricionales requieren cumplir una serie de condiciones para ser considerados como tales:

1. Que se haya demostrado su biodisponibilidad, esto es, que la dosis administrada alcance su diana o, lo que es lo mismo, que se absorban y lleguen a los tejidos deseados en cantidades adecuadas.

2. Que las dosis de vitaminas y minerales de los suplementos respeten las CDR (cantidades diarias recomendadas), ya que no se trata de dosis farmacológicas, sino de dosis nutricionales.

3. Que existan estudios que avalen su eficacia, valorando y cuantificando los cambios en el ór- gano al que se destinan. La actividad biológica de los distintos principios activos de los suplementos nutricionales se ha estudiado en múltiples ámbitos como son la prevención cardiovascular con los fitoesteroles y omega-3, entre otros, o la salud ósea con los componentes de la soja, por poner algunos ejemplos.

4. Que la seguridad esté avalada por los estudios científicos.

\section{LOS SUPLEMENTOS NUTRICIONALES Y LA PIEL}

Es sabido que la dieta tiene un papel importante en el desarrollo de muchos trastornos dermatológicos (fig. 1) y que los suplementos nutricionales son necesarios en ocasiones como complementos terapéuticos (por ejemplo, el cinc en la acrodermatitis enteropática, o los suplementos de aceite de pescado ricos en ácidos grasos poliinsaturados o PUFA [del inglés: poly-unsaturated fatty acids] en la psoriasis) o como agentes adicionales en estrategias del campo de la estética. Estos últimos son los que admiten mayor controversia.

Que los principios activos de los suplementos nutricionales llegan a la piel —esto es, su biodisponibilidad - se ha demostrado en muchos de ellos en diferentes trabajos. En cierto modo, todos los estudios que han comprobado la eficacia de un suplemento nutricional son congruentes con una biodisponibilidad adecuada. Un ejemplo de alta evidencia es el trabajo de Blume-Peytavi et al. ${ }^{1}$, quienes demostraron en un estudio aleatorio controlado con placebo y doble ciego que el licopeno y el betacaroteno administrados por vía oral llegaban a la piel humana, conduciendo a un enriquecimiento cutáneo en dichos elementos.

A la hora de valorar la eficacia de un suplemento nutricional en el campo de la belleza, hay que juzgar no solo los resultados globales en el aspecto de la piel y la prevención de enfermedades cutáneas, sino también la acción específica de cada uno de sus componentes.

En este último aspecto, cobran especial importancia las vitaminas, imprescindibles en múltiples 


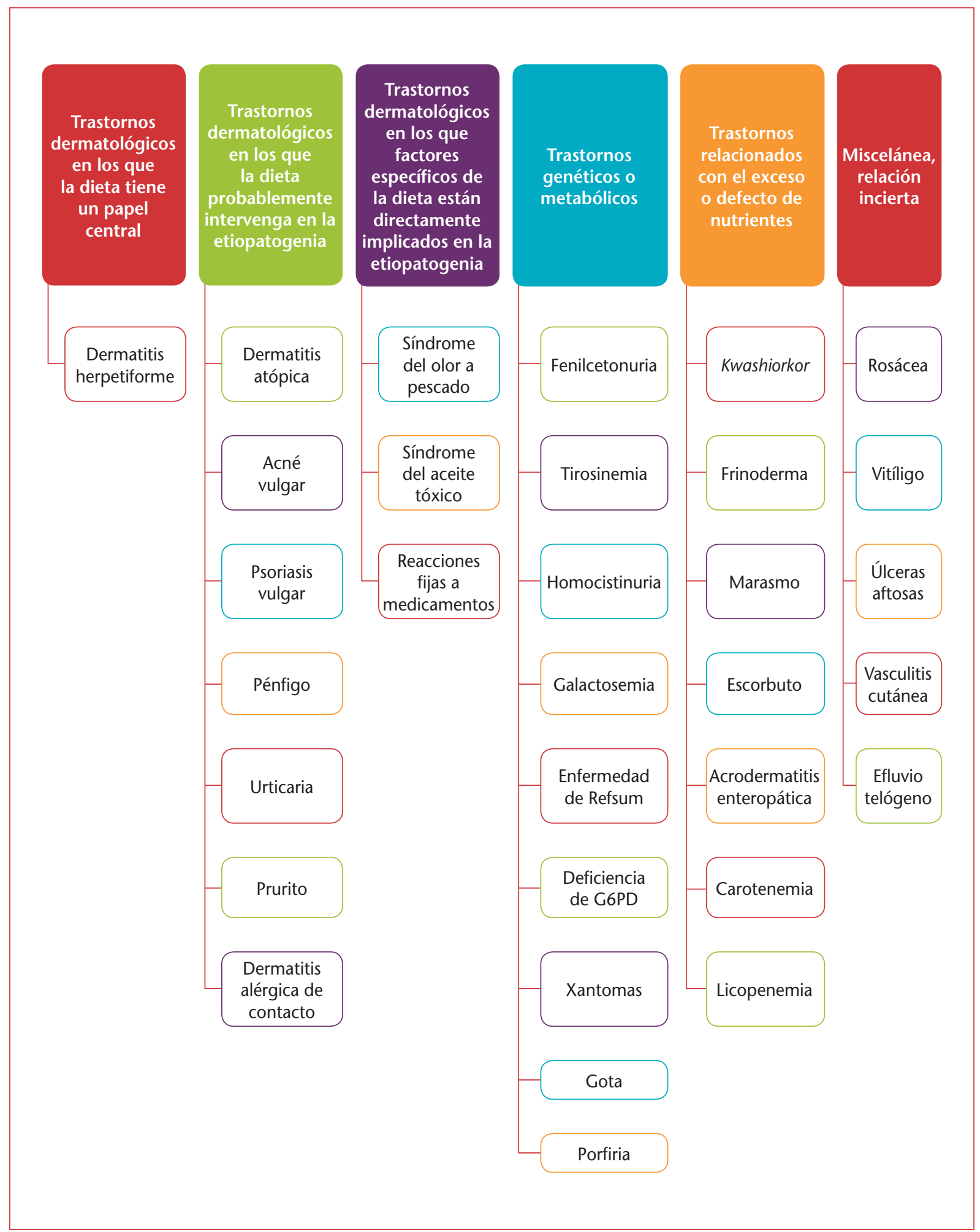

Figura 1. Algunos trastornos dermatológicos relacionados con la alimentación.

G6PD: glucosa 6-fosfato-deshidrogenasa (glucose-6-phosphate dehydrogenase). 
funciones bioquímicas esenciales para la correcta funcionalidad del ser humano. Los dos tipos de vitaminas (liposolubles e hidrosolubles) necesitan una ingesta regular, pero las hidrosolubles, por su naturaleza transitoria, tienen mayor riesgo de deficiencia, sobre todo, cuando existe un alto consumo de alcohol, en los síndromes de malabsorción, dietas veganas estrictas, trastornos de la alimentación y otros estados de desnutrición².

En este grupo, se encuentra la vitamina $\mathrm{C}$ o ácido ascórbico, potente antioxidante, cofactor, complemento enzimático, sustrato en una gran variedad de reacciones y procesos metabólicos, sintetizada por múltiples plantas y animales, pero no por los primates, debido a la falta de la enzima L-gluconolactona-oxidasa, necesaria para su producción. En la piel, actúa en la formación del colágeno a través del procolágeno, la hidroxiprolina y la hidroxilisina como pasos previos. También se ha demostrado que estimula la síntesis de procolágeno de tipo I en cultivos de fibroblastos humanos ${ }^{3}$, mejorando el envejecimiento cutáneo. Por otra parte, el ácido ascórbico también parece influir en la síntesis de elastina degradada, disminuyendo la fabricación de esta proteína por los fibroblastos. Esta acción resulta beneficiosa para la piel fotoexpuesta, ya que reduciría la acumulación de elastina degradada que se produce en el fotoenvejecimiento. Incluso se le han demostrado propiedades fotoprotectoras ${ }^{4}$ : algunos trabajos clásicos y otros recientes confirman que la suplementación oral con vitamina $\mathrm{C}$ disminuye el estrés oxidativo cutáneo producido por la exposición a la radiación ultravioleta, aumentando de forma significativa a las ocho semanas de una ingesta de $500 \mathrm{mg}$ /día el contenido de vitamina $\mathrm{C}$ en la piel ${ }^{5}$.

Junto a estos y otros estudios que avalan la acción de la vitamina $\mathrm{C}$ como promotora del colágeno dérmico, reductora de la elastina y antioxidante, existen otros que evalúan la mejora global de la piel, como el publicado en $2015^{6}$, en el que se administró durante 180 días un suplemento oral que contenía vitamina C (27 mg), extracto de semillas de uva $(13,75 \mathrm{mg})$, cinc $(2 \mathrm{mg})$ y extracto de tomate $(14,38 \mathrm{mg})$ a voluntarios sanos, encon- trando una mejora del envejecimiento de la piel objetivado con resultados estadísticamente significativos: aumento de la hidratación de la piel (nivel de significación estadística $[p]<0,05)$, incremento de la densidad del ultrasonido dérmico $(p<0,001)$ y reducción del $\mathrm{pH}(p<0,05)$. Además, en la valoración por parte de los sujetos del estudio y de los investigadores, se describía una mejora del eritema, la hidratación, la luminosidad y la apariencia general de la piel del rostro.

Las vitaminas hidrosolubles del grupo $\mathrm{B}$ comprenden ocho tipos: vitamina $\mathrm{B}_{1}$ (tiamina), $\mathrm{B}_{2}$ (riboflavina), $\mathrm{B}_{3}$ (niacinamida), $\mathrm{B}_{5}$ (ácido pantoténico), $\mathrm{B}_{6}$ (piridina), $\mathrm{B}_{7}$ (biotina, vitamina $\mathrm{H}$ ), $\mathrm{B}_{9}$ (folato) y vitamina $B_{12}$ (cianocobalamina), teniendo todas ellas un papel significativo en diferentes procesos en el metabolismo celular. Quizá la biotina es la que con mayor frecuencia se encuentra incluida en los suplementos nutricionales dirigidos a la piel.

La deficiencia de biotina es poco común, aunque se refiere en numerosos trabajos casi siempre asociada a trastornos genéticos del metabolismo. La utilidad de su suplementación, además de en estos casos, está avalada para el tratamiento de trastornos del cabello y de las uñas. También las embarazadas y los fumadores necesitan una mayor dosis, beneficiándose de esta suplementación ${ }^{7}$. Hay que tener en cuenta que, según diversos estudios publicados en 2017, la biotina así administrada interfiere en determinadas pruebas de laboratorio (fundamentalmente, en la determinación de los niveles de hormonas tiroideas, de troponina y otras), pudiendo provocar diagnósticos equivocados, por lo que es preciso suspender la suplementación antes de realizar pruebas de laboratorio ${ }^{8}$.

Uno de los antioxidantes con importante acción en la mejora de la piel es la astaxantina, carotenoide de color rojo anaranjado liposoluble ${ }^{9}$ presente en algunas bacterias y algas. Tiene, además, propiedades antiinflamatorias, antiapoptóticas e inmunomoduladoras ${ }^{10}$. Desempeña un papel fundamental en la hidratación cutánea, favoreciendo la expresión y la actividad de la acuaporina-3 o, lo 
que es lo mismo, la vía elemental de la hidratación ${ }^{11}$. Por esta acción, está indicada especialmente en el tratamiento de la piel seca y la piel envejecida, además de en variadas enfermedades con manifestaciones cutáneas ${ }^{12}$.

Un componente de los suplementos nutricionales que más controversia ha suscitado es el colágeno oral, sobre todo, por la duda que se alberga acerca de su biodisponibilidad en la piel. Un estudio publicado en $2020^{13}$ ha venido a aportar evidencia científica, por su diseño y desarrollo, en un ensayo triple ciego, aleatorizado, y controlado con placebo. El suplemento ingerido durante 12 semanas contenía péptidos de colágeno como ingrediente principal, y vitamina $\mathrm{C}$, vitamina $\mathrm{E}$, biotina y cinc. La microscopia de barrido láser confocal demostró una mejora significativa de la estructura del colágeno de la piel facial y de otras zonas tratadas frente a la del placebo, resultados congruentes con parámetros clínicos como la elasticidad, las arrugas y la uniformidad, valorados por corneometría (hidratación), cutometría (elasticidad), réplicas de silicona con mediciones ópticas in vivo tridimensionales (rugosidad) y ecografía de piel (densidad). Otro estudio análogo de $2019^{14}$ aportaba resultados similares.

\section{CONCLUSIONES}

Estos y otros ejemplos hablan a favor de los suplementos nutricionales siempre que su indicación sea procedente. La multiplicidad de principios activos simultánea —-sinergismo- potencia la asimilación y su consiguiente eficacia.

Aunque es cierto que una alimentación adecuada es la base de la salud y la prevención de la enfermedad, determinados nutrientes de acción favorable sobre el órgano cutáneo a menudo no alcanzan la cantidad suficiente a través la dieta como para poder ejercer sus efectos beneficiosos. El empobrecimiento de las tierras de cultivo en algunos minerales, el cambio en la alimentación animal, y la dieta occidental -en muchos casos, favorecedora de procesos inflamatorios y prooxidantes- hacen imprescindibles ciertas interven- ciones de suplementación innecesarias hace unas décadas.

No obstante, la preocupación por la seguridad debe estar presente tanto en los prescriptores como en los usuarios: las interacciones con medicamentos, los riesgos de teratogenicidad o la sobredosis son algunos de los «fantasmas que cruzan por el cielo» de los suplementos nutricionales ${ }^{15}$.

En conclusión, los suplementos nutricionales pueden conseguir resultados beneficiosos sobre la piel de individuos sanos y enfermos, siempre que estén bien indicados y personalizados.

\section{BIBLIOGRAFÍA}

1. Blume-Peytavi U, Rolland A, Darvin ME, Constable A, Pineau I, Voit C, et al. Cutaneous lycopene and beta-carotene levels measured by resonance Raman spectroscopy: high reliability and sensitivity to oral lactolycopene deprivation and supplementation. Eur J Pharm Biopharm. 2009;73(1):187-94.

2. Lykstad J, Sharma S. Biochemistry, Water Soluble Vitamins. En: StatPearls [Internet]. Treasure Island: StatPearls Publishing; 2020.

3. Chung JH, Youn SH, Kwon OS, Cho KH, Youn Jl, Eun HC. Regulations of collagen synthesis by ascorbic acid, transforming growth factor-beta and interferon-gamma in human dermal fibroblasts cultured in three-dimensional collagen gel are photoaging- and aging-independent. J Dermatol Sci 1997;15(3):188-200.

4. Shapira N. Nutritional approach to sun protection: a suggested complement to external strategies. Nutr Rev. 2010;68(2): 75-86.

5. McArdle F, Rhodes LE, Parslew R, Jack CIA, Friedmann PS, Jackson MJ. UVR-induced oxidative stress in human skin in vivo: effects of oral vitamin $C$ supplementation. Free Radic Biol Med. 2002;33(10):1355-62.

6. Costa A, Samartin Pegas Pereira E, Assumpção EC, Calixto Dos Santos FB, Ota FS, de Oliveira Pereira M, et al. Assessment of clinical effects and safety of an oral supplement based on marine protein, vitamin C, grape seed extract, zinc, and tomato extract in the improvement of visible signs of skin aging in men. Clin Cosmet Investig Dermatol. 2015;8:319-28.

7. Bistas KG, Tadi P. Biotin. En: StatPearls [Internet]. Treasure Island: StatPearls Publishing; 2020.

8. Waqas B, Wu A, Yim E, Lipner SR. A survey-based study of physician practices regarding biotin supplementation. J Dermatolog Treat. 2020:1-2. [En prensa].

9. Mohammadi S, Barzegari A, Dehnad A, Barar J, Omidi Y. Astaxanthin protects mesenchymal stem cells from oxidative stress by direct scavenging of free radicals and modulation of cell signaling. Chem Biol Interact. 2021;333:109324.

10. Chang MX, Xiong F. Astaxanthin and its effects in inflammatory responses and inflammation-associated diseases: recent advances and future directions. Molecules. 2020;25(22): 5342.

11. Ikarashi N, Kon R, Nagoya C, Ishikura A, Sugiyama Y, Takahashi J, et al. Effect of astaxanthin on the expression and activity of 
la opinión del experto | Suplementos nutricionales y piel

aquaporin-3 in skin in an in-vitro study. Life (Basel). 2020; 10(9):193.

12. Ng QX, De Deyn MLZQ, Loke W, Foo NX, Chan HW, Yeo WS. Effects of astaxanthin supplementation on skin health: a systematic review of clinical studies. J Diet Suppl. 2020:1-14. [En prensa].

13. Laing S, Bielfeldt S, Ehrenberg C, Wilhelm KP. A dermonutrient containing special collagen peptides improves skin structure and function: a randomized, placebo-controlled, triple-blind trial using confocal laser scanning microscopy on the cosme- tic effects and tolerance of a drinkable collagen supplement. J Med Food. 2020;23(2):147-52.

14. Bolke L, Schlippe G, Gerß J, Voss W. A collagen supplement improves skin hydration, elasticity, roughness, and density: results of a randomized, placebo-controlled, blind study. Nutrients. 2019;11(10):2494.

15. Pérez-Sánchez AC, Burns EK, Pérez VM, Tantry EK, Prabhu S, Katta R. Safety concerns of skin, hair and nail supplements in retail stores. Cureus. 2020;12(7):e9477. 